\title{
¿Qué sabemos sobre los hombres que maltratan a su pareja? Una revisión sistemática
}

\author{
Mercedes Carrasco-Portiño, ${ }^{1}$ Carmen Vives-Cases, ${ }^{1}$ \\ Diana Gil-González ${ }^{1}$ y Carlos Álvarez-Dardet ${ }^{1}$
}

Forma de citar Carrasco-Portiño M, Vives-Cases C, Gil-González D, Álvarez-Dardet C. ¿Qué sabemos sobre los hombres que maltratan a su pareja? Una revisión sistemática. Rev Panam Salud Publica. 2007;22(1):55-63.

RESUMEN Objetivos. Analizar los artículos publicados en revistas científicas entre los años 2000 y 2005 enfocados en los hombres que maltratan a sus parejas e identificar las características de los estudios empíricos cuantitativos.

Métodos. Revisión sistemática de los artículos publicados entre enero de 2000 y junio de 2005 que se centran en el tema de los hombres que maltratan a sus parejas. La búsqueda se realizó en las bases de datos EconLit, Embase, Eric, Francis, Índice Médico Español, ISI Web of Knowledge -Web of Science y Current Contents-, Medline, Psicodoc, PsycInfo y Sociological Abstracts.

Resultados. Se identificaron 944 estudios, de los cuales $61(6,5 \%)$ se centraron en el análisis. Treinta y cuatro $(55,7 \%)$ se publicaron en revistas especializadas en la violencia y solamente $4(6,6 \%)$ se publicaron en revistas de Ciencias Médicas; 47 (77,0\%) procedian de instituciones norteamericanas —especialmente de los Estados Unidos de América (70,5\%) - y solamente 1 (1,6\%) artículo procedía de América Latina (Puerto Rico). De los 29 artículos que utilizaron métodos de análisis cuantitativo, 19 (65,5\%) abordaron las causas o factores de riesgo relacionados con la violencia doméstica como eje central de su investigación y 10 $(34,5 \%)$ centraron su atención en intervenciones dirigidas a los agresores.

Conclusiones. Se encontraron pocos estudios científicos que abordaran la etiología de la violencia contra la mujer en la pareja y los encontrados utilizaron diseños epidemiológicos sin el suficiente poder explicativo para establecer relaciones causales. Los estudios que se centran en el problema desde un punto de vista curativo no presentan suficiente evidencia acerca de la eficacia de los programas dirigidos a maltratadores. No se encontraron estudios publicados en revistas de salud pública. La carencia de mayor información científica puede estar impidiendo que se tomen decisiones politicas informadas y se pongan en marcha intervenciones más eficaces.

Palabras clave Violencia contra la mujer, maltrato conyugal, revisión, violencia doméstica.

1 Universidad de Alicante, Área de Medicina Preventiva y Salud Pública, Alicante, España. La correspondencia se debe dirigir a Mercedes Carrasco Portiño, Universidad de Alicante, Área de Medicina Preventiva y Salud Pública, Campus San Vicente de Raspeig, A.P. 99, C.P. 03080, Alicante, España. Correo electrónico: mercedes.carrasco@ua.es
La violencia contra las mujeres en su vida de pareja constituye un abuso contra los derechos humanos y uno de los problemas de salud pública fundamentales y crecientes en todo el mundo. Al mismo tiempo es una manifestación extrema de la desigualdad de género que afecta a las mujeres, según el Informe Mundial sobre la Violencia y la Salud, de la Organización Panamericana de la Salud (1). 
La violencia del compañero íntimo es un problema que afecta y pone en riesgo a la salud de las mujeres (2). Se ha estimado que entre $13 \%$ y $61 \%$ de las mujeres ha sufrido algún tipo de maltrato físico por parte de su pareja en algún momento de su vida (3). Además, en la literatura científica se alude a un posible "efecto acumulativo" de las diversos tipos de maltrato, de manera que el impacto en la salud es mayor en las mujeres que experimentan abusos físicos, sexuales y psicológicos que en las que experimentan un solo tipo de abuso $(4,5)$.

La gravedad de este problema en la salud y en el bienestar de las mujeres se pone de manifiesto también en el impacto económico y social que conlleva (6-8). No obstante, la evidencia empírica existente sobre las intervenciones en torno a la violencia contra la mujer en la pareja y sus resultados es todavía insuficiente (9), especialmente con relación al origen del problema. Son pocas las investigaciones enfocadas en los hombres que maltratan a sus parejas (10-12).

Se observa una mayor tendencia a estudiar la situación de las mujeres afectadas por la violencia de pareja que a los perpetradores de esa violencia (13). Este enfoque podría limitar el conocimiento acerca de la causalidad de este problema, ya que al hacer énfasis en por qué las mujeres afectadas no huyen de su agresor o no lo denuncian se transforma un inmenso problema social en un asunto de carácter privado en el que toda la responsabilidad recae en la víctima (14). Es necesario que las investigaciones sobre la violencia contra la mujer en la pareja también contemplen la figura del agresor como causa del conflicto, lo que contribuiría a conocer mejor la etiología del problema y a desarrollar intervenciones basadas en evidencias científicas, tanto preventivas como de rehabilitación (11).

Los objetivos de este estudio fueron analizar los artículos publicados en revistas científicas entre los años 2000 y 2005 enfocados en los hombres que maltratan a sus parejas e identificar las características de los estudios empíricos cuantitativos.

\section{MATERIALES Y MÉTODOS}

Se realizó una revisión sistemática de las publicaciones científicas que abordan el tema de los hombres que maltratan a sus parejas. Para la búsqueda de artículos científicos publicados entre enero de 2000 y junio de 2005 se consultaron diez de las bases de datos de Ciencias de la Salud y Ciencias Sociales de mayor visibilidad en Europa y las Américas: EconLit, Embase, Eric, Francis, Índice Médico Español, ISI Web of Knowledge - Web of Science y Current Contents-, Medline, Psicodoc, PsycInfo y Sociological Abstracts. La decisión de limitar la búsqueda al período 2000-2005 fue arbitraria y se basó en la intención de examinar los datos más recientes.

Se utilizaron las palabras clave en las bases datos que contaban con descriptores o vocabulario controlado (tesauros), como Medline, Eric y Psycoinfo. En las bases de datos que no contaba con esta herramienta se utilizaron las siguientes palabras clave como palabras naturales: "battered women", "battered female", "domestic violence", "partner abuse" y "spousal abuse" en sus combinaciones con las palabras "perpetrator", "aggressor" y "offender". Se utilizaron también los términos en español "violencia doméstica", "violencia de género" y "violencia contra la mujer" combinados con "maltratador" $\mathrm{y}$ "agresor".
En las bases de datos que lo permitían se utilizaron campos de interrogación específicos, como los siguientes: título del artículo, nombre de los autores, fuente del artículo primario, idioma del documento original (inglés o español), tipo de documento (sólo con resumen disponible), año de publicación (2000-2005) y descriptores.

Para la selección de los artículos se estableció como criterio de inclusión básico el hecho de que en sus objetivos o hipótesis se tomara como objeto de estudio algún aspecto relacionado con los hombres que maltratan a sus parejas. Se excluyeron los estudios sobre violencia contra la mujer en la pareja $u$ otro tipo de violencia centrados exclusivamente en las víctimas, ya fueran estudios sobre la etiología del problema, su prevalencia e impacto, o intervenciones. Además, se excluyeron los editoriales, los estudios de revisión, las ponencias o comunicaciones a congresos y las recensiones de libros (cuadro 1).

La información se captó mediante un protocolo que contemplaba las siguientes variables:

a) Aspectos generales de los artículos: año de publicación (de 2000 a 2005) y disciplina de la revista (especializada en violencia, psicología o psiquiatría, ciencias sociales, jurídicas o criminalísticas, o ciencias médicas).

b) Aspectos relacionados con la autoría de los estudios: sexo del autor que aparece en primer lugar en las fir-

CUADRO 1. Resultados de la búsqueda de artículos publicados sobre la violencia contra la mujer en la pareja y de la selección de los artículos centrados en el agresor

\begin{tabular}{lrr}
\hline \multicolumn{1}{c}{ Artículos identificados } & No. & $\%$ \\
\hline Incluidos en el análisis & 61 & 6,5 \\
Excluidos del análisis & 883 & 93,5 \\
$\quad$ Estudios sobre víctimas de violencia doméstica y su impacto & 401 & 42,5 \\
$\quad \begin{array}{l}\text { Descripción o evaluación de intervenciones en víctimas de la violencia } \\
\quad \text { contra las mujeres }\end{array}$ & 228 & 24,2 \\
$\quad \begin{array}{l}\text { Estudios transversales basados en la población general sobre violencia } \\
\quad \text { en general }\end{array}$ & 140 & 14,8 \\
$\quad \begin{array}{l}\text { Estudios centrados en la aplicación del tamizaje de la violencia contra } \\
\quad \text { las mujeres en la pareja }\end{array}$ & 75 & 7,9 \\
$\quad \begin{array}{l}\text { Investigaciones sobre la etiología de la violencia contra las mujeres en } \\
\quad \text { la pareja centradas en factores de riesgo de las víctimas }\end{array}$ & 35 & 3,7 \\
$\quad$ Revisiones y recensiones de libros & 4 & 0,4 \\
$\quad$ Total & 944 & 100,0 \\
\hline
\end{tabular}


mas - a partir de su nombre completo-; país del primer autor o autora - por áreas geográficas y países-; disciplina a que se dedicaba el primer autor o autora, según su profesión o actividad laboral en el momento de firmar el artículo -medicina general, medicina forense, medicina preventiva, psiquiatría, otras ciencias de la salud, legislación, educación, psicología o sociología, ciencias de la vida-; y tipo de institución responsable de la investigación - universidad, centro de reclusión, centro de investigación, instituto de justicia, servicios de salud o no identificado.

c) Aspectos metodológicos de los estudios: tipo de estudio realizado -empírico o teórico-, tipo de metodología aplicada - cuantitativa, cualitativa o ambas—, tipo de diseño de los estudios - de cohorte, de casos y controles, transversales, series de casos o cualitativos-, objeto principal del estudio - intervención, etiología, percepción de los agresores $\mathrm{u}$ otro-, tipo de intervención -tratamiento, rehabilitación, coerción $\mathrm{u}$ otro- $\mathrm{y}$ factores de riesgo analizados en los estudios etiológicos - antecedentes de violencia en la infancia, consumo de alcohol, bajo nivel socioeconómico, papel en función del género o trastornos psíquicos (15).

Para validar el protocolo de recogida de información de los artículos se realizó un estudio de concordancia simple. Las codificadoras (MCP y DGG) analizaron de forma independiente una muestra de diez artículos, con un alto nivel de concordancia (90\%). Para el análisis de los datos se utilizó el programa estadístico SPSS versión 11.5 (16).

\section{RESULTADOS}

Se identificaron 944 estudios que contemplan entre sus objetivos o hipótesis a los hombres que maltratan a su pareja. Después de su revisión, se excluyeron $883(93,5 \%)$ artículos por tratarse de estudios centrados en las víctimas de la violencia doméstica

CUADRO 2. Características generales de los artículos seleccionados $(n=61)$

\begin{tabular}{|c|c|c|}
\hline Variables y sus categorías & No. & $\%$ \\
\hline \multicolumn{3}{|l|}{ Año de publicación } \\
\hline 2000 & 8 & 13,1 \\
\hline 2001 & 20 & 32,8 \\
\hline 2002 & 7 & 11,5 \\
\hline 2003 & 12 & 19,7 \\
\hline 2004 & 14 & 23,0 \\
\hline \multicolumn{3}{|l|}{ Especialidad de la revista científica } \\
\hline Violencia & 34 & 55,7 \\
\hline Psicología y psiquiatría & 15 & 24,6 \\
\hline Ciencias sociales & 6 & 9,8 \\
\hline Ciencias médicas & 4 & 6,6 \\
\hline Ciencias jurídicas y criminalísticas & 2 & 3,3 \\
\hline \multicolumn{3}{|l|}{ Sexo del/a primer/a autor/a } \\
\hline Mujer & 23 & 37,7 \\
\hline Hombre & 38 & 62,3 \\
\hline \multicolumn{3}{|l|}{ Origen de las publicaciones } \\
\hline América del Norte & 47 & 77,0 \\
\hline Estados Unidos & 43 & 70,5 \\
\hline Canadá & 4 & 6,6 \\
\hline América Latina & 1 & 1,6 \\
\hline Puerto Rico & 1 & 1,6 \\
\hline Europa & 10 & 16,4 \\
\hline Reino Unido & 4 & 6,6 \\
\hline España & 6 & 9,8 \\
\hline Asia (Israel) & 2 & 3,3 \\
\hline Oceanía (Nueva Zelanda) & 1 & 1,6 \\
\hline \multicolumn{3}{|l|}{ Disciplina del primer autor } \\
\hline Psicología & 31 & 50,8 \\
\hline Ciencias médicas & 11 & 18,0 \\
\hline Sociología & 10 & 16,4 \\
\hline Psiquiatría & 6 & 9,8 \\
\hline Ciencias jurídicas & 3 & 4,9 \\
\hline
\end{tabular}

(cuadro 1). Por tanto, en la revisión sistemática se analizaron $61(6,5 \%)$ artículos (17-77).

\section{Características generales de los estudios}

La mayoría de los artículos analizados se publicaron en los años 2001 (20 artículos; 32,8\% del total), 2003 (12 artículos; $19,7 \%$ ) y en 2004 (14 artículos; 23,0\%) (cuadro 2).

De los artículos analizados, 34 $(55,7 \%)$ se publicaron en revistas especializadas en la violencia y solamente 4 $(6,6 \%)$ se publicaron en revistas de ciencias médicas y $2(3,3 \%)$ en revistas dedicadas a ciencias jurídicas y criminalísticas (cuadro 2).

Con respecto a la autoría de los trabajos analizados, predominaron los hom- bres, con 38 artículos $(62,3 \%)$ frente a 23 artículos $(37,7 \%)$ de mujeres como primeras autoras. Del los 61 artículos, 47 $(77,0 \%)$ procedían de instituciones norteamericanas - especialmente de los Estados Unidos de América (70,5\%) - y solamente $1(1,6 \%)$ artículo procedía de América Latina (Puerto Rico) (cuadro 2).

La mayoría $(31 ; 50,8 \%)$ de los primeros autores estaba vinculada con Psicología como especialidad, 11 (18\%) con diversas disciplinas de las Ciencias Médicas y solamente $3(4,9 \%)$ de ellos estaban relacionados con las Ciencias Jurídicas (cuadro 2).

\section{Diseño de los estudios}

En general, 34 (55,7\%) de los estudios analizados eran empíricos (17-50) y 27 $(44,3 \%)$ eran teóricos (51-77). Cinco 
CUADRO 3. Estudios etiológicos y de intervención sobre violencia contra las mujeres en la pareja, según el componente de la pareja en que se centraron $(n=384)$

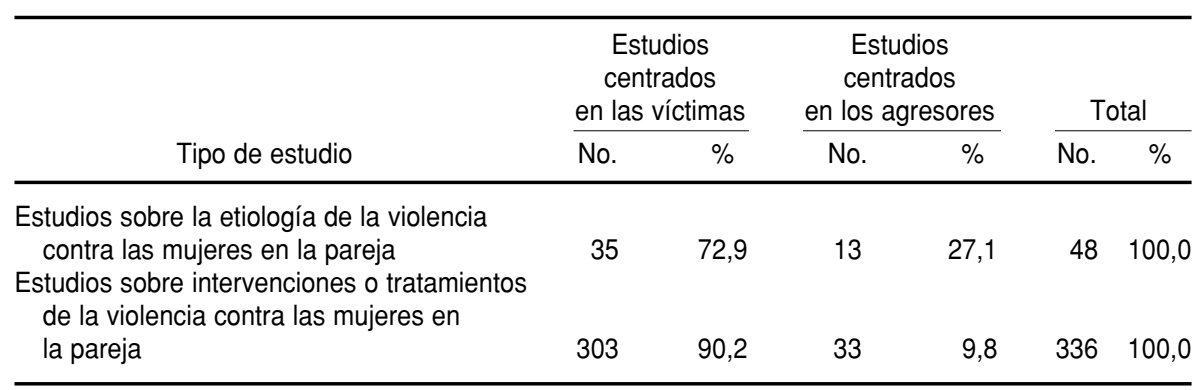

$(14,7 \%)$ de los estudios empíricos utilizaron métodos cualitativos en su análisis $(22,34,40,45,48)$. De los 29 artículos que utilizaron métodos de análisis cuantitativo, 19 (65,5\%) abordaron las causas o factores de riesgo relacionados con la violencia doméstica como eje central de su investigación (17-20, 23, $29,32,33,35,36,38,39,42-44,46,47,49$, $50)$; de ellos, $14(73,7 \%)$ utilizaron un diseño transversal $(17,19,29,33,35,36$, $38,39,42-44,46,47,49), 3$ (15,8\%) eran estudios experimentales $(18,20,23) \mathrm{y}$ solamente $2(10,5 \%)$ eran de casos y controles $(32,50)$.

Del total de estudios cuantitativos, 10 $(34,5 \%)$ centraron su atención en intervenciones dirigidas a los agresores (21, $24-28,30,31,37,41)$, de ellos $7(70,0 \%)$ tenían un diseño transversal $(21,24-26$, $30,31,41), 2(20,0 \%)$ eran estudios de cohorte $(27,37)$ y $1(10,0 \%)$ tenía un diseño cuasiexperimental (28).

De los 384 artículos localizados mediante las combinaciones de palabras clave establecidas al inicio del estudio, que abordaban aspectos etiológicos o analizaban intervenciones, 336 (87,5\%) analizaban algún tipo de intervención contra la violencia de la compañera íntima; de ellos $303(90,2 \%)$ se referían exclusivamente a las víctimas y solamente $33(9,8 \%)$ se centraron en los agresores. Por su parte, de los 384 artículos sobre etiología e intervenciones, $48(12,5 \%)$ trataban sobre factores de riesgo asociados con la violencia contra la mujer, y de estos, 35 (72,9\%) se centraron en las víctimas, mientras que solamente $13(27,1 \%)$ se enfocaron en la conducta y el perfil de los agresores (cuadro 3).

\section{Estudios sobre la etiología de la violencia del compañero íntimo}

Los 19 artículos que tenían un diseño epidemiológico de corte transversal $(17-20,23,29,32,33,35,36,38,39$, $42-44,46,47,49,50)$ permitieron explorar las características de las poblaciones estudiadas, tanto de los agresores como de sus parejas, ya que analizan algunas características demográficas - como la edad, el nivel educacional, el tipo de empleo, los ingresos económicos, el número de hijos, la etnia- y personales - como la situación marital de la pareja, el consumo de alcohol y drogas y el número de arrestos por violencia- de los sujetos de estudio. En los estudios transversales cuyo objetivo era describir las características conductuales, se observaron diversas escalas de clasificación acordes con el enfoque del trabajo (si fue víctima o testigo de violencia familiar o social en la infancia o en la adolescencia), el tipo de personalidad, el tipo de violencia de que fue víctima (sexual, física o psicológica), el tipo de violencia que comete, el nivel de dependencia interpersonal, etc.)

Dada la importancia que tiene el diseño epidemiológico para establecer las posibles causas de la violencia contra la mujer en la pareja y su relación con determinados factores de riesgo, se deben destacar, por encima de otros diseños, los resultados y conclusiones de los estudios de casos y controles, ya que permiten hacer inferencias acerca de las causas de la violencia (cuadro 4). Rosenbaum y Leisring encontraron una asociación estadísticamente signi- ficativa entre el haber sido víctima de la violencia durante la infancia y el posterior comportamiento agresivo de una parte de los hombres estudiados (32). Cohen y colaboradores demostraron que el déficit en la capacidad de expresión verbal y la conducta impulsiva son factores que predispusieron a los hombres a cometer actos violentos (50). Finalmente, estudios experimentales en que se utilizó una sustancia panicogénica (lactato sódico) para analizar las posibles causas de la violencia contra la mujer encontraron que la conducta violenta responde a una exacerbada reacción de los agresores frente al miedo, el pánico y la rabia (18, $20,23)$, que a su vez es potenciada por alteraciones en las estructuras neurológicas de agresores consumidores de alcohol (23).

\section{Estudios de intervención}

En los estudios centrados en intervenciones dirigidas a los agresores con un diseño epidemiológico transversal se pueden ver las características demográficas de los agresores incorporados a programas de rehabilitación, tanto voluntariamente como por orden judicial $(21,24,25,26,30,31,41)$. Algunos de esos estudios tienen un enfoque etiológico y analizan las características individuales y conductuales de los agresores, además de brindar una valiosa información acerca de los diversos tipos de agresores $(26,31)$. Además, en ellos se describen los programas de intervención, su duración y costos, los fundamentos teóricos de las técnicas de rehabilitación $(21,24,25,41)$ y -en menor medida- la percepción de los agresores con respecto a diversos programas de intervención $(30,41)$.

Según los resultados de un estudio de cohorte (37), los hombres que terminaron algún programa de intervención cometieron menos agresiones contra su pareja que los que se retiraron antes de terminarlo (cuadro 5). Scott y Wolfe (27) evaluaron la aplicabilidad del modelo transteórico del cambio de comportamiento abusivo y concluyeron que las intervenciones dirigidas a hombres que maltratan a sus 
CUADRO 4. Características de los estudios epidemiológicos sobre la etiología de la violencia contra la mujer en la pareja

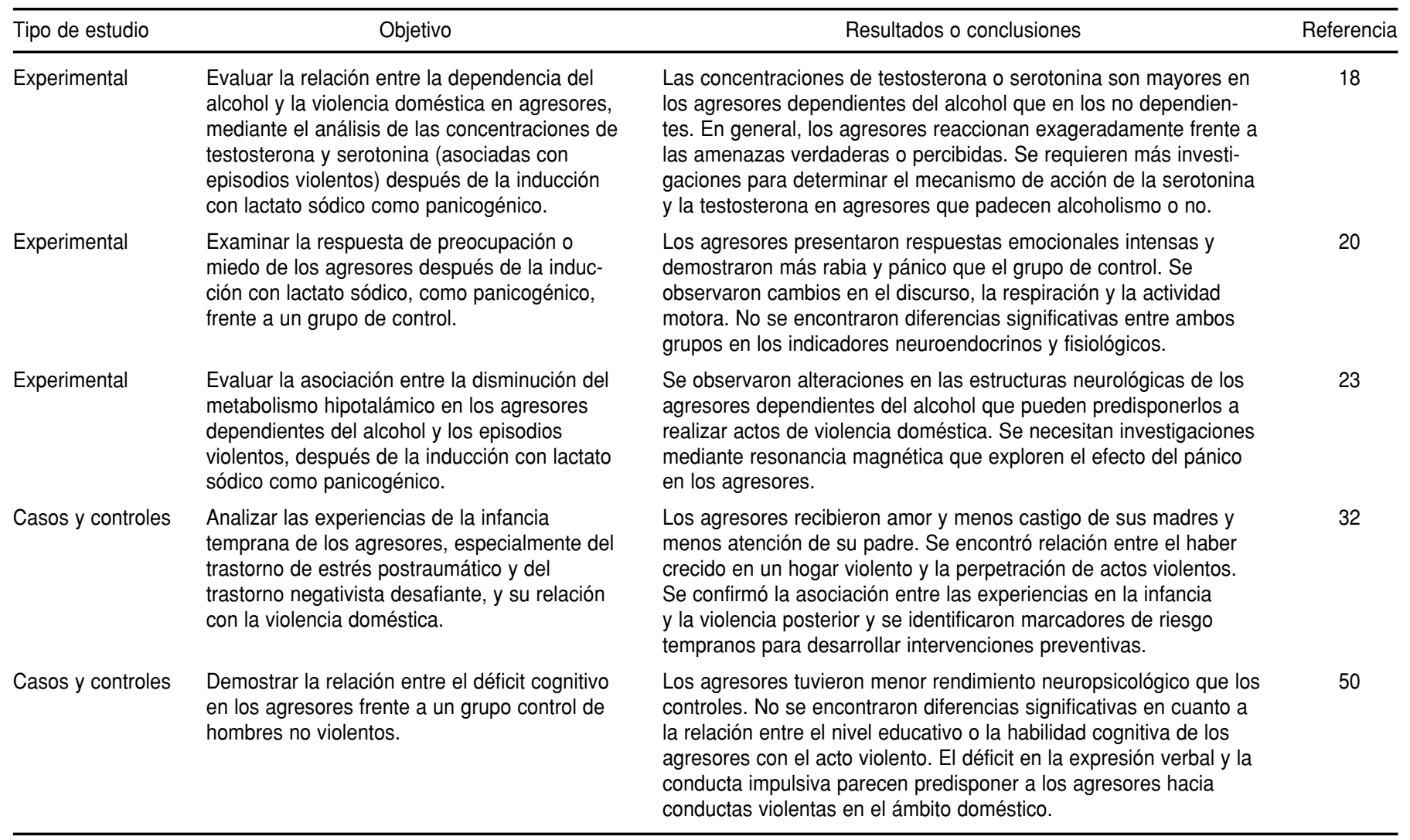

CUADRO 5. Características de los estudios epidemiológicos de violencia contra las mujeres en la pareja centrados en intervenciones o tratamientos para agresores

\begin{tabular}{|c|c|c|c|}
\hline Tipo de estudio & Objetivo & Resultados o conclusiones & Referencia \\
\hline De cohorte & $\begin{array}{l}\text { Evaluar un programa para agresores de sus } \\
\text { parejas (derivados al programa por orden } \\
\text { judicial) en cuatro ciudades de EE.UU. y } \\
\text { determinar su eficacia en la reducción de la } \\
\text { reincidencia. }\end{array}$ & $\begin{array}{l}\text { Los agresores que permanecieron en el programa } 3 \text { meses o más } \\
\text { reincidieron significativamente menos que los que abandonan el } \\
\text { programa en los cuatro programas analizados, a pesar de las } \\
\text { diferencias de los programas en cuanto a extensión y servicios. }\end{array}$ & 37 \\
\hline De cohorte & $\begin{array}{l}\text { Identificar los factores que influyen en que los } \\
\text { agresores de sus parejas completen el trata- } \\
\text { miento de rehabilitación, mediante el modelo } \\
\text { transteórico de Prochaska (estrategia para } \\
\text { pronosticar el cambio en agresores). }\end{array}$ & $\begin{array}{l}\text { A las } 10 \text { semanas de tratamiento, los hombres en etapa de precon- } \\
\text { templación mostraron menos progresos en empatía, comunicación } \\
\text { y comportamiento agresivo que los que estaban en la etapa de } \\
\text { meditación y de acción. Después de } 10 \text { semanas se observaron } \\
\text { avances positivos en todos los participantes. }\end{array}$ & 27 \\
\hline
\end{tabular}

parejas no deben ser uniformes y que, aunque este modelo es una de las pocas estrategia útiles en este sentido, no garantiza que todos los agresores que ingresan al programa de intervención corrijan su actuación.

\section{DISCUSIÓN}

Los artículos que se centran en los hombres que maltratan a sus parejas son todavía escasos en la literatura científica en comparación con los estu- dios enfocados en las víctimas de maltratos. El número de publicaciones dedicadas a analizar los casos de violencia del compañero íntimo parece ir en aumento en los últimos años, aunque este tema no se ha llegado a con- 
solidar aún como una línea de investigación epidemiológica. Los estudios epidemiológicos son de gran utilidad para identificar los factores de riesgo, tanto individuales como del entorno, ya que contribuyen a desarrollar estrategias de intervención bien informadas. Los estudios que tratan este problema con un enfoque curativo no presentan suficiente evidencia empírica sobre la eficacia de los programas dirigidos a agresores. A pesar de que la violencia contra la mujer en la pareja es un problema de salud pública, no se han encontrado estudios publicados en revistas de esta especialidad.

Se encontraron pocos estudios científicos que abordaran la etiología de la violencia contra la mujer en la pareja y los encontrados utilizaron diseños epidemiológicos sin el suficiente poder explicativo para establecer relaciones causales. Esto confirma lo encontrado por otros autores en una revisión que analizaba la relación existente entre el consumo de alcohol y la conducta violenta de los hombres contra sus parejas (78). Esta carencia dificulta el desarrollo adecuado de intervenciones y estrategias de prevención basadas en la evidencia.

Los estudios sobre la etiología de este problema que utilizaron diseños más complejos abordaron únicamente los factores neurológicos o psiquiátricos que pueden predisponer a los agresores a cometer actos violentos $(18,20,23)$. Sin embargo, dada la complejidad del problema, para lograr un enfoque más integrador se tendrían que tomar también en cuenta los aspectos relacionados con el individuo y su entorno, ya que pueden influir en el comportamiento violento de los hombres frente a su pareja. En relación con esto último, un estudio reciente confirmó la relación que hay entre la desigualdad de género y el riesgo de las mujeres de padecer o morir por la violencia de su pareja (79).

Según algunos autores, la situación de la violencia contra las mujeres en América Latina y el Caribe es especialmente alarmante (1). Sin embargo -y a pesar de que la mayoría de los artículos identificados provienen de la Región de las Américas-, no se encon- traron investigaciones promovidas en o dirigidas hacia los países latinoamericanos y caribeños, con excepción de un estudio que describe el escenario de la violencia doméstica en Puerto Rico y ofrece una aproximación a un modelo teórico multidimensional para comprender y explicar la violencia doméstica (74). Resulta especialmente interesante la descripción de las iniciativas de prevención de la violencia doméstica en ese país y de las intervenciones realizadas. No obstante, según los propios autores, las acciones dirigidas a hombres que incurren en conductas de maltrato de su pareja íntima no constituyen un objetivo prioritario dentro de las políticas portorriqueñas y no se cuenta con los recursos económicos necesarios para llevarlas a cabo (74).

Según algunas revisiones, el aporte de los grupos de investigación estadounidenses sobre la violencia contra la mujer es considerable, tanto con relación a las víctimas como a los agresores $(78,80,81)$. Los resultados obtenidos en el presente trabajo confirman esta apreciación, en especial los aportes de Gondolf y White relacionados con los programas de intervención en los Estados Unidos y, más específicamente, en los programas centrados en los agresores con trastornos de la personalidad (37, 38, 39). Los trabajos de David George relacionados con la etiología de la violencia contra las mujeres $(18,20,23)$ son también de gran importancia.

Las investigaciones sobre intervenciones dirigidas a agresores no solo deben dirigir su atención hacia la reducción de la violencia, sino también a identificar con exactitud qué hombres pueden ser más susceptibles de beneficiarse con los tratamientos disponibles. En este sentido hay evidencias de que los hombres que participan de forma voluntaria en programas de rehabilitación terminan exitosamente su tratamiento con mayor frecuencia que los que participan obligados por una orden judicial (42). Asimismo, según los resultados analizados, los hombres que asisten a un mayor número de sesiones de un programa de rehabilitación para agresores recaen menos en actos de abuso hacia sus parejas y mejoran sus habilidades de comunicación y de empatía $(27,37,42)$. Sin embargo, aún no se conoce lo suficiente acerca de los factores que favorecen estos resultados, como la voluntariedad y el cumplimiento de los programas de rehabilitación.

A pesar del esfuerzo investigativo realizado para identificar alternativas rehabilitadoras eficientes dirigidas a los hombres que maltratan a sus parejas íntimas, todavía no se dispone de datos robustos que indiquen cómo se puede reducir o erradicar la reincidencia en actos de violencia contra la mujer en la pareja. Antes de poder identificar intervenciones eficaces dirigidas a la rehabilitación de esos hombres es necesario acumular una mayor evidencia empírica sobre este tema, especialmente con relación a la evaluación de los programas dirigidos a reducir las conductas violentas de los hombres contra sus parejas (82).

Los resultados obtenidos en este estudio no son exhaustivos debido a que las bases de datos consultadas no abarcan a todas las publicaciones y a que puede existir mucha información relevante en la llamada literatura gris. Una limitación de este estudio es que la búsqueda bibliográfica se realizó a mediados del año 2005, lo que permitió analizar solamente la información publicada hasta junio de ese año. No se encontró el número esperado de artículos acerca de la violencia contra la mujer y sus agresores en países en desarrollo de América Latina, el Caribe, África y Asia, donde esta situación puede ser tan o más grave que la descrita en este trabajo. El hecho de que las investigaciones sobre los hombres que maltratan a sus parejas provienen principalmente de países desarrollados - en primer lugar de los Estados Unidos- puede sesgar estos resultados. Sin embargo, se exploraron las bases de datos europeas y americanas más importantes, tanto en las áreas de Ciencias de la Salud como de Ciencias Sociales, lo que reduce considerablemente esa limitación.

Los resultados encontrados demuestran que son pocos los artículos publicados entre los años 2000 y 2005 que estudian a los hombres que maltratan a sus parejas. La carencia de mayor in- 
formación científica puede estar impidiendo que se tomen decisiones políticas informadas y se pongan en marcha intervenciones más eficaces.

Se recomienda aumentar el número de investigaciones enfocadas en los hombres que maltratan a sus parejas íntimas, en particular las dirigidas a estudiar las causas de esa conducta violenta, los tratamientos rehabilitado- res y las medidas de prevención eficaces. Se deben identificar las poblaciones de agresores más susceptibles de beneficiarse con los tratamientos disponibles y la forma de reducir las tasas de reincidencias de la violencia contra la mujer en la pareja. Es necesario que las investigaciones tomen en cuenta tanto los elementos del agresor como de su entorno, a fin de explicar mejor los factores que llevan a esa conducta violenta. En la Región de las Américas se deben emprender investigaciones sobre la violencia contra las mujeres en la pareja, especialmente en los países de América Latina y el Caribe, de manera que pueda enriquecerse y particularizarse más la visión de este grave problema en los contextos sociales y culturales específicos de cada país.

\section{REFERENCIAS}

1. Organización Panamericana de la Salud. Informe Mundial sobre la Violencia y la Salud. Ed. 2002. Washington, D.C.: OPS; 2002. Hallado en http://www.paho.org/Spanish/ AM/PUB/Violencia_2003.htm. Acceso el 2 de mayo de 2007.

2. Hegarty K, Gunn J, Chondros P, Small R. Association between depression and abuse by partners of women attending general practice: descriptive, cross sectional survey. Br Med J. 2004;328:621-4.

3. World Health Organization. Multi-Country Study on Women's Health and Domestic Violence against Women: initial results on prevalence, health outcomes and women's responses. Geneva: WHO; 2005. Hallado en http://www.who.int/gender/violence/who _multicountry_study/en/index.html. Acceso el 1 de mayo de 2007.

4. Wingood GM, DiClemente RJ, Raj A. Adverse consequences of intimate partner abuse among women in non-urban domestic violence shelters. Am J Prev Med. 2000;19:270-5.

5. Romito P, Molzan TJ, De Marchi M. The impact of current and past interpersonal violence on women's mental health. Soc Sci Med. 2005;60:1717-27.

6. National Center for Injury Prevention and Control. Cost of intimate partner violence against women in the United States. Atlanta, Georgia, EUA: Centers for Disease Control and Prevention; 2003. Hallado en http:// www.cdc.gov/ncipc/pub-res/ipv_cost/IPV Book-Final-Feb18.pdf. Acceso el 2 de mayo de 2007.

7. Walby S. The cost of domestic violence. En: Queen Mary's School of Medicine and Dentistry. Interventions to reduce violence and promote the physical and psychosocial wellbeing of women who experience partner violence: a systematic review of controlled evaluations. London: Barts and The London Queen Mary's School of Medicine and Dentistry; 2005. Hallado en http://www.dh.gov.uk/ assetRoot/04/12/74/26/04127426.pdf. Acceso el 2 de mayo de 2007.

8. Instituto Andaluz de la Mujer. Los costes sociales y económicos de la violencia contra las mujeres en Andalucía. Sevilla, España: Instituto Andaluz de la Mujer; 2004.

9. Wathen $\mathrm{CN}$, MacMillan HL. Intervention for violence against women: scientific review. J Am Med Assoc. 2003;289(5):589-600.
10. Lutenbacher M, Cohen A, Mitzel J. Do we really help? Perspective of abused women. Public Health Nurs. 2003;20(1):56-64.

11. Pan-American Health Organization. Program on Women Health and Development. Progress in the eradication of violence against women. Washington, D.C.: PAHO; 1998.

12. Ramsay J, Rivas C, Feder G. Interventions to reduce violence and promote the physical and psychosocial well-being of women who experience partner violence: a systematic review of controlled evaluations. London: Barts and The London Queen Mary's School of Medicine and Dentistry; 2005. Hallado en http:// www.dh.gov.uk/assetRoot/04/12/74/26/ 04127426.pdf. Acceso el 2 de mayo de 2007.

13. Browne A. Violence against women by male partners. Prevalence, outcomes, and policy implications. Am J Psychol. 1993;48:1077-87.

14. Renzetti C, Edleson J, Bergen R. Sourcebook on violence against women. California: Sage Publications; 2001.

15. Heise L. Violence against women. An integrated, ecological framework. Violence Against Women. 1998;4(3):262-90.

16. Bryman A, Cramer D. Quantitative data analysis with SPSS release 10 for Windows: a guide for social scientists. London: Editorial Routledge; 2001.

17. Monson C, Langhinrichsen-Rohling J. Sexual and nonsexual dating violence perpetration: Testing an integrated perpetrator typology. Violence and Victims. 2002;17(4):403-28.

18. George D, Umhau J, Phillips M, Emmela D, Ragan P, Shoaf S, et al. Serotonin, testosterone and alcohol in the etiology of domestic violence. Psychiatric Research. 2001;104(1): 27-37.

19. Langhinrichsen-Rohling J, Huss MT, Ramsey S. The clinical utility of batterer typologies. J Fam Violence. 2000;15(1):37-53.

20. George D, Hibbeln J, Ragan P, Umhau J, Phillips M, Doty L, et al. Lactate-induced rage and panic in a select group of subject who perpetrate acts of domestic violence. Biol Psychiatry. 2000;47:804-12.

21. Eckhardt C, Babcock J, Homack S. Partner assaultive men and the stages and processes of change. J Fam Violence. 2004;19(2):81-93.

22. Workey K, Walsh S, Lewis K. An examination of parenting experiences in male perpetrators of domestic violence: a qualitative study. Psychol Psychotherapy. 2004;77(1):35-54.
23. George D, Rawlings R, Williams W, Phillips M, Fong, G, Kerich M, et al. A select group of perpetrators of domestic violence: evidence of decreased metabolism in the right hypothalamus and reduced relationships between cortical/subcortical brain structures in position emission tomography. Psychiatry Neuroimaging. 2004;130(1):11-25.

24. Rosenberg M, Dutton D, Sonkin D. Voices from the group: domestic violence offenders experience of intervention. Intimate violence: contemporary treatment innovations. J Aggress Maltreat Trauma. 2003;7(1-2):305-17.

25. Schumacher J, Fals-Stewart W, Leonard K. Domestic violence treatment referrals for men seeking alcohol treatment. J Subst Abuse Treat. 2003;24(3):279-83.

26. Buttell F, Pike C. Investigating predictors of treatment attrition among court-ordered batterers. J Soc Serv Resh. 2002;28(4):53-68.

27. Scott K, Wolfe D. Readiness to change as a predictor of outcome in batterer treatment. J Consult Clin Psychol. 2003;71(5):879-89.

28. Snow Jones A, D'Agostino R, Gondolf E, Heckert A. Assessing the effect of batterer program completion on reassault using propensity scores. J Interpers Violence. 2004;19 (9):1002-20.

29. Babcock J, Green Ch, Webb S, Graham K. A second failure to replicate the Gottman et al. (1995) typology of men who abuse intimate partners and possible reasons why. J Fam Psychol. 2004;18(2):396-400.

30. Buchbinder E, Eisikovits Z. Between normality and deviance. The breakdown of batterers identity following police intervention. J Interpers Violence. 2004;19(4):443-67.

31. Yarbrough D, Blanton P. Socio-demographic indicators of intervention program completion with the male court-referred perpetrator of partner abuse. J Criminal Justice. 2000;28: 517-26.

32. Rosenbaum A, Leisring PA. Beyond power and control: towards an understanding of partner abusive men. J Comp Fam Stud. 2003; 34(1):7-22.

33. Dobash E, Dobash R, Cavanagh K, Lewis R Not an ordinary killer-just an ordinary guy. When men murder and intimate woman partner. Violence Against Women. 2004;10(6): 577-605.

34. Cavanagh K, Dobash E, Dobash R, Lewis R. "Remedial Work": men's strategic responses 
to their violence against intimate female partners. Sociology. 2001;35(3):695-714.

35. Follingstad D, DeHart D. Defining psychological abuse of husbands toward wives: contexts, behaviors, and typologies. J Interpers Violence. 2000;15(9):891-920.

36. Lucero M, Middleton K, Finch W, Valentine S. An empirical investigation of sexual harassers: toward a perpetrator typology. Hum Relat. 2003;56(12):1461-83.

37. Gondolf E. A 30-month follow-up courtreferred batterers in four cities. Int J Offender Ther Comp Criminol. 2000;44(1):111-28.

38. Gondolf E, White R. Batterer program participants who repeatedly reassault: psychopathic tendencies and other disorders. J Interpers Violence. 2001;16(4):361-80.

39. White R, Gondolf E. Implications of personality profiles for batterer treatment. J Interpers Violence. 2000;15(5):467-88.

40. Laviolette A, Geffner R, Rosenbaum A. Batterer's treatment: observations from the trenches. J Aggress Maltreat Trauma. 2001; 5(2):45-56.

41. Bennett L, Vincet N, Geffner R, Rosenbaum A. Standards for batterer programs: a formative evaluation of the Illinois Protocol. J Aggress Maltreat Trauma. 2001;5(2):181-97.

42. Rosenbaum A, Gearan P, Ondovic C, Geffner R. Completion and recidivism among courtand self-referred batterers in a psychoeducational group treatment program: implications for intervention and public policy. J Aggress Maltreat Trauma. 2001;5(2):199-220.

43. Coben J, Friedman D. Health care by perpetrators of domestic violence. J Emerg Med. 2002;22(3):313-7.

44. Buttell $\mathrm{F}$, Jones $\mathrm{C}$. Interpersonal dependency among court-ordered domestic violence offenders: a descriptive analysis. J Fam Violence. 2001;16(4):375-84.

45. Goodrum S, Umberson D, Anderson K. The batterers view of the self and others in domestic violence. Sociol Inq. 2001;71(2):221-40.

46. Burgess A, Harner H, Baker T, Hartman C. Batterer's stalking patterns. J Fam Violence. 2001;16(3):309-21.

47. Matud Aznar M, Carballeira Abella M, Marrero Quevedo R, Aguilera Ávila L, Moraza Pulla O, Pérez Trujillo N. Características sociodemográficas y conductuales de los agresores a su pareja: un análisis a través del informe de las víctimas. Psicopatol Clin Legal Forense. 2002;2(2):5-22.

48. Winstok Z, Eisikovoits Z, Gelles R. Structure and dynamics of escalation from the batterer's perspective. Fam Soc. 2002;83(2):129-41.

49. Bennett L, Goodman L, Dutton MA. Risk assessment among batterers arrested of domestic assault. Violence Against Women. 2000; 6(11):1190-203.

50. Cohen R, Brumm V, Zawachi TM, Paul R, Sweet L, Rosembaum A. Impulsivity and verbal deficits associated with domestic violence. J Int Neuropsychol Soc. 2003;9:760-90.
51. Dutton D. The neurobiology of abandonment homicide. Aggress Violent Behaviour. 2002;7: 407-21.

52. Koss M. Blame, shame, and community: justice responses to violence against women. Am Psychol. 2000;55(11):1332-43.

53. Prentky R. Can sex offender classification inform typologies of male batterers? A response to Holtzworth-Munroe and Meehan. J Interpers Violence. 2004;19(12):1405-11.

54. Rivett M, Rees A. Dancing on a razor's edge: systematic group work with batterers. J Fam Ther. 2004;26(2):142-66.

55. Diefenbeck C. Group therapy for male batterers: comparison of cognitive-behavioural and object relations approaches. J Psychosoc Nurs Ment Health Serv. 2003;41(10):18-25.

56. Sonkin D, Dutton D. Treating assaultive men from an attachment perspective. Intimate violence: contemporary treatment innovations. J Aggress Maltreat Trauma. 2003;7(1-2):105-33.

57. Kiyoshk R, Dutton D, Sonkin D. Integrating spirituality and domestic violence treatment: treatment of aboriginal men. Intimate violence: contemporary treatment innovations. J Aggress Maltreat Trauma. 2003;7(1-2):237-56.

58. Corvo K, Johnson P. Vilification of the "batterer": how blame shapes domestic violence policy and interventions. Aggress Violent Behavior. 2003;8:259-81.

59. Sonkin D, Liebert D. The assessment of courtmandated perpetrators of domestic violence. J Aggress Maltreat Trauma. 2003;6(2):3-36

60. Holtzworth-Munroe A, Geffner R, Rosenbaum A. Standards for batterer programs: how can research inform our decisions? J Aggress Maltreat Trauma. 2001;5(2):165-80.

61. Murphy C. Toward empirically based standards for abuser intervention: the Maryland model. J Aggress Maltreat Trauma. 2001;5(2): 249-64.

62. Geffner R, Rosenbaum A. Domestic violence offenders: treatment and intervention standards. J Aggress Maltreat Trauma. 2001;5(2): $1-9$.

63. Saunders D. Developing guidelines for domestic violence offender programs: what can we learn from related fields and current research? J Aggress Maltreat Trauma. 2001;5(2): 235-48.

64. Campbell J. Safety planning based on lethality assessment for partner of batterers in intervention programs. J Aggress Maltreat Trauma. 2001;5(2):129-43.

65. Tolman R. An ecological analysis of batterer intervention program standards. J Aggress Maltreat Trauma. 2001;5(2):221-33.

66. Hamberger K. Musings of state standards committee chair. J Aggress Maltreat Trauma. 2001;5(2):265-86.

67. Mankowski E, Haaken J, Silvergleid C. Collateral damage: an analysis of the achievements and unintended consequences of batterer intervention programs and discourse. J Fam Violence. 2002;17(2):167-84.
68. Rosenbaum A, Leisring P, Geffner R. Group intervention programs for batterers. J Aggress Maltreat Trauma. 2001;5(2):57-71.

69. Rosenbaum A, Geffner R. Future directions in mandated standards for domestic violence offenders. J Aggress Maltreat Trauma. 2001;5(2): 287-93.

70. Gelles R. Standards for program for men who batter? Not yet. J Aggress Maltreat Trauma. 2001;5(2):11-20.

71. Romans S, Poore M, Martin J. The perpetrators of domestic violence. Med J Aust. 2000; 173(9):484-8.

72. López García E. La figura del agresor en la violencia de género: características personales e intervención. Papeles Psicol. 2004;88:31-8.

73. Echeburúa E, de Corral P, FernándezMontalvo J, Amor PJ. ¿Se puede y debe tratar psicológicamente a los hombres violentos contra la pareja? Papeles Psicol. 2004:88; 10-8.

74. Ortiz A, García E. Violencia doméstica: modelo de análisis y programas de intervención de agresores. Cuad Trab Soc. 2003;16:193-215.

75. Castellano Arroyo M, Lachica López E, Molina Rodríguez A, Villanueva de la Torre H. Violencia contra la mujer. El perfil del agresor: criterios de valoración del riesgo. Cuad Med Forense. 2004:35;15-28.

76. Echeburúa Odriozola E, Fernández-Montalvo J, de la Cuesta JL. Articulación de medidas penales y de tratamiento psicológico en los hombres violentos en el hogar. Psicopatol Clin Legal Forense. 2001;1(2):19-31.

77. Barea Payueta C. El maltratador: ¿enfermo o delincuente? Rev FMC. 2004;11(6):300-5.

78. Gil-González D, Vives-Cases C, ÁlvarezDardet C, Latour-Pérez J. Alcohol and intimate partner violence: do we have enough information to act? Eur J Public Health. 2006; 16(3):278-84.

79. Vives-Cases C, Álvarez-Dardet C, CarrascoPortiño M, Torrubiano J. El impacto de la desigualdad de género en la violencia del compañero íntimo en España. Gac Sanit. 2007; 21(3):242-6.

80. Feldman CM. Childhood precursors of adult interpartner violence. Clin Psychol Scie Pract. 1997;4:307-34.

81. Römkens R. Protecting prosecution: exploring the powers of law in an intervention program for domestic violence. Violence Against Women. 2006;12(2):160-86.

82. Silvergleid CS, Mankowski ES. How batterer intervention programs work: participant and facilitator accounts of processes of change. J Interpers Violence. 2006;21(1):139-59.

Manuscrito recibido el 1 de junio de 2006. Aceptado para publicación, tras revisión, el 26 de junio de 2007. 
ABSTRACT Objectives. To analyze articles published in scientific journals from 2000 to 2005 that specifically focus on men who abuse their female partner, and to identify characteristics that the quantitative empirical studies have in common.

\section{What do we know about men who abuse their female partner? A systematic review}

Methods. A systematic review of articles published from January 2000 to June 2005 on the topic of men who abuse their female partner was conducted by searching the following databases: EconLit, Embase, Eric, Francis, Índice Médico Español, ISI Web of Knowledge (Web of Science and Current Contents), Medline, Psicodoc, PsycInfo, and Sociological Abstracts.

Results. Of the 944 studies identified, victim-specific articles were eliminated, and $61(6.5 \%)$ were analyzed. Of these, violence-related journals had published $34(55.7 \%)$; medical sciences journals had published only 4 (6.6\%). North American institutions had published 47 of the 61 articles $(77.0 \%)$, most of which were from the United States of America (70.5\%), and only 1 article (1.6\%) came from Latin America (Puerto Rico). Of the 29 articles that employed quantitative analysis, 19 (65.5\%) examined the etiology of and risk factors related to domestic violence, and 10 (34.5\%) focused on interventions geared toward the aggressor.

Conclusions. Few scientific studies were found that tackled the causes of violence against the female partner, and those that did used epidemiological methods that fell short of quantifying the causes of partner violence. Studies that approach the issue from a curative angle do not provide enough evidence on the effectiveness of programs aimed at abusers. No articles were found in public health journals. The absence of scientific literature on this topic could be impeding informed policy-making and hindering efforts to put more effective intervention programs in place.

Key words

Battered women, spouse abuse, domestic violence, review literature.

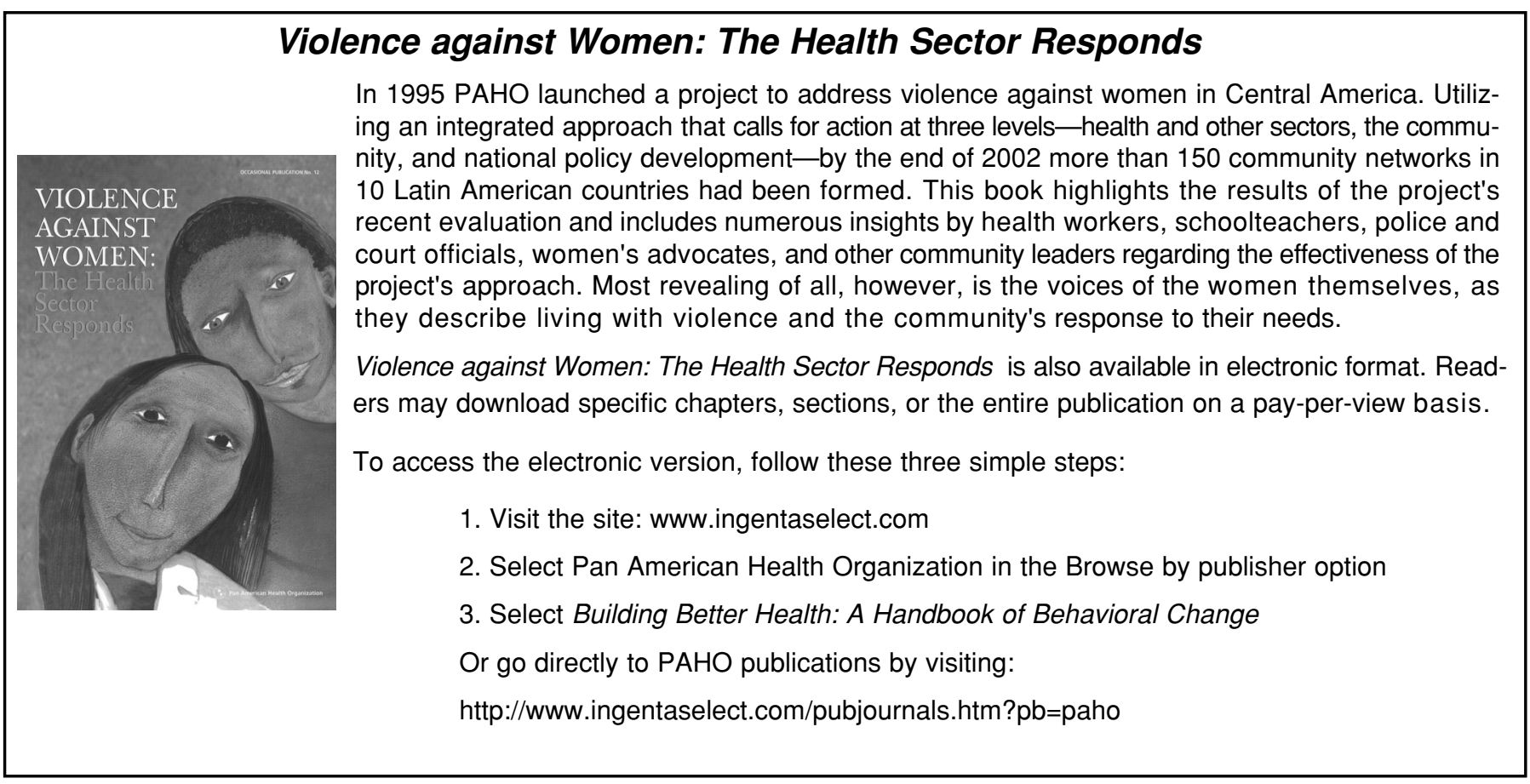

